\title{
NA48/62 latest results
}

\author{
Radoslav Marchevski*† \\ Johannes Gutenberg University, Mainz, Germany \\ E-mail: rmarchevduni-mainz.de
}

The NA62 experiment at the CERN SPS recorded in 2007 a large sample of $K^{+} \rightarrow \mu^{+} v_{\mu}$ decays. A peak search in the missing mass spectrum of this decay is performed. In the absence of observed signal, the limits obtained on $\mathscr{B}\left(K^{+} \rightarrow \mu^{+} v_{h}\right)$ and on the mixing matrix element $\left|U_{\mu 4}\right|$ are reported. The upgraded NA62 experiment started data taking in 2015 . About $5 \times 10^{11} K^{+}$decays have been recorded so far to measure the branching ratio of the $K^{+} \rightarrow \pi^{+} v \bar{v}$ decay. Preliminary results from the $K^{+} \rightarrow \pi^{+} v \bar{v}$ analysis based on about $5 \%$ of the 2016 statistics are reported.

The 15th International Conference on Flavor Physics \& CP Violation

5-9 June 2017

Prague, Czech Republic

\footnotetext{
*Speaker.

${ }^{\dagger}$ On behalf of the NA62 Collaboration: R. Aliberti, F. Ambrosino, R. Ammendola, B. Angelucci, A. Antonelli, G. Anzivino, R. Arcidiacono, M. Barbanera, A. Biagioni, L. Bician, C. Biino, A. Bizzeti, T. Blazek, B. Bloch-Devaux, V. Bonaiuto, M. Boretto, M. Bragadireanu, D. Britton, F. Brizioli, M.B. Brunetti, D. Bryman, F. Bucci, T. Capussela, A. Ceccucci, P. Cenci, V. Cerny, C. Cerri, B. Checcucci, A. Conovaloff, P. Cooper, E. Cortina Gil, M. Corvino, F. Costantini, A. Cotta Ramusino, D. Coward, G. D’Agostini, J. Dainton, P. Dalpiaz, H. Danielsson, N. De Simone, D. Di Filippo, L. Di Lella, N. Doble, B. Dobrich, F. Duval, V. Duk, J. Engelfried, T. Enik, N. Estrada-Tristan, V. Falaleev, R. Fantechi, V. Fascianelli, L. Federici, S. Fedotov, A. Filippi, M. Fiorini, J. Fry, J. Fu, A. Fucci, L. Fulton, E. Gamberini, L. Gatignon, G. Georgiev, S. Ghinescu, A. Gianoli, M. Giorgi, S. Giudici, F. Gonnella, E. Goudzovski, C. Graham, R. Guida, E. Gushchin, F. Hahn, H. Heath, T. Husek, O. Hutanu, D. Hutchcroft, L. Iacobuzio, E. Iacopini, E. Imbergamo, B. Jenninger, K. Kampf, V. Kekelidze, S. Kholodenko, G. Khoriauli, A. Khotyantsev, A. Kleimenova, A. Korotkova, M. Koval, V. Kozhuharov, Z. Kucerova, Y. Kudenko, J. Kunze, V. Kurochka, V.Kurshetsov, G. Lanfranchi, G. Lamanna, G. Latino, P. Laycock, C. Lazzeroni, M. Lenti, G. Lehmann Miotto, E. Leonardi, P. Lichard, L. Litov, R. Lollini, D. Lomidze, A. Lonardo, P. Lubrano, M. Lupi, N. Lurkin, D. Madigozhin, I. Mannelli, G. Mannocchi, A. Mapelli, F. Marchetto, R. Marchevski, S. Martellotti, P. Massarotti, K. Massri, E. Maurice, M. Medvedeva, A. Mefodev, E. Menichetti, E. Migliore, E. Minucci, M. Mirra, M. Misheva, N. Molokanova, M. Moulson, S. Movchan, M. Napolitano, I. Neri, F. Newson, A. Norton, M. Noy, T. Numao, V. Obraztsov, A. Ostankov, S. Padolski, R. Page, V. Palladino, C. Parkinson, E. Pedreschi, M. Pepe, M. Perrin-Terrin, L. Peruzzo, P. Petrov, F. Petrucci, R. Piandani, M. Piccini, J. Pinzino, I. Polenkevich, L. Pontisso, Yu. Potrebenikov, D. Protopopescu, M. Raggi, A. Romano, P. Rubin, G. Ruggiero, V. Ryjov, A. Salamon, C. Santoni, G. Saracino, F. Sargeni, V. Semenov, A. Sergi, A. Shaikhiev, S. Shkarovskiy, D. Soldi, V. Sougonyaev, M. Sozzi, T. Spadaro, F. Spinella, A. Sturgess, J. Swallow, S. Trilov, P. Valente, B. Velghe, S. Venditti, P. Vicini, R. Volpe, M. Vormstein, H. Wahl, R. Wanke, B. Wrona, O. Yushchenko, M. Zamkovsky, A. Zinchenko.
} 


\section{Heavy neutrino searches in $K^{+} \rightarrow \mu^{+} v_{\mu}$ decays}

With the increasing evidences that neutrinos have non-zero masses, it is necessary to extend the Standard Model (SM) to accommodate them. The Neutrino Minimal Standard Model ( $v$ MSM) [U] solves this problem by adding 3 massive right-handed neutrinos to the SM. Effective vertices with the $\mathrm{W}^{ \pm} ; \mathrm{Z}$ and the $\mathrm{SM}$ leptons can be built, with a mixing matrix $U$ describing the mixing between the heavy neutrinos and the SM neutrinos. The SM neutrinos then acquire masses through the see-saw mechanism. For heavy neutrinos with masses below the kaon mass, limits on their mixing matrix elements can be placed by searching for peaks in the missing mass spectrum of $K^{ \pm}$ decays [[]]. This analysis focuses on the $K^{+} \rightarrow \mu^{+} v_{\mu}$ decay to search for heavy neutrino in the mass range $300-375 \mathrm{MeV} / \mathrm{c}^{2}$. Strong limits of the order of $10^{-8}$ (up to $300 \mathrm{MeV} / \mathrm{c}^{2}$ ) and $10^{-6}$ (up to $330 \mathrm{MeV} / \mathrm{c}^{2}$ ) on the element $\left|U_{\mu 4}\right|^{2}$ are already set by stopped kaon experiments [3], 团]. The heavy neutrino acceptance drops quickly above $375 \mathrm{MeV} / \mathrm{c}^{2}$, because it is approaching the limit $m_{K}-m_{\mu}=388 \mathrm{MeV} / c^{2}$. The following assumptions are made: the heavy neutrinos decay only to SM particles, and $\left|U_{\mu 4}\right|^{2}<10^{-4}$, such that the mean free path of heavy neutrinos in the mass range considered is longer than $10 \mathrm{~km}$. Their decay can then be neglected as the probability of decaying in the detector of decay volume is below $1 \%$. Because only the production process is looked at, the limits scale linearly with the kaon flux.

\subsection{The NA62 experimental setup in 2007}

During the data-taking campaign of 2007, the NA62 experiment collected a large sample of kaon decays in-flight. The high-efficiency single track trigger was designed to collect $K^{ \pm} \rightarrow e^{ \pm} v_{e}$, $K^{ \pm} \rightarrow \mu^{ \pm} v_{\mu}$ decays, aiming at a test of the lepton universality in the kaon sector [四]. Part of the $K^{ \pm} \rightarrow \mu^{ \pm} v_{\mu}$ sample recorded is used to search for the production of heavy neutrino. The beam line, described in detail in [目] , was designed to provide simultaneous $K^{+}$and $K^{-}$beams. They were extracted from the $400 \mathrm{GeV} / \mathrm{c}$ SPS proton beam impinging on a $40 \mathrm{~cm}$ long beryllium target. The final beam momentum of $(74.0 \pm 1.4) \mathrm{GeV} / c$ was selected using a system of dipole magnets and a momentum-defining slit incorporated into a beam dump. The beams were focused and collimated before entering the $114 \mathrm{~m}$ long cylindrical vacuum tank containing the fiducial decay volume. The beam contained mainly pions but included approximately $6 \%$ of kaons. The momenta of the charged particles, produced by the decayed kaons, were measured by a magnetic spectrometer housed in a tank filled with helium at approximately atmospheric pressure. It was composed of four drift chambers (DCHs) and a dipole magnet located between the second and third chambers. A hodoscope (HOD) composed of two planes of plastic scintillator was placed after the spectrometer to provide precise timing of the charged particles and generate fast trigger signals for the low-level trigger. A $127 \mathrm{~cm}$ thick quasi-homogeneous electromagnetic calorimeter filled with liquid krypton ( $\mathrm{LKr}$ ) was located downstream, followed by a muon veto system (MUV) which consisted of three planes of scintillator orthogonal to the beam axis, each one preceded by a $80 \mathrm{~cm}$ thick iron wall.

The main trigger condition for selecting the $K^{+} \rightarrow \mu^{+} N\left(v_{\mu}, v_{h}\right)$ required at least one coincidence of hits in the two HOD planes, and bounds on the hits multiplicity in the DCH. This trigger line was down-scaled by a factor 150 . To obtain the purest sample, only the data taking periods 


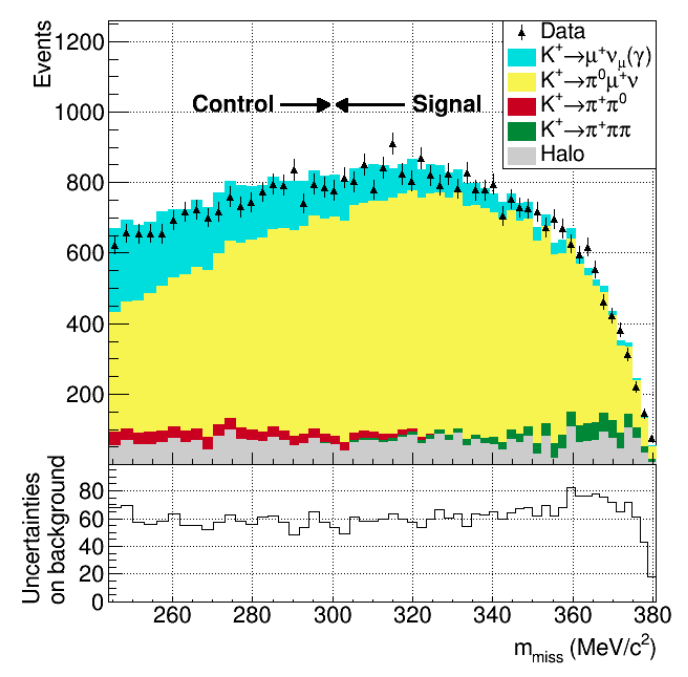

(a)

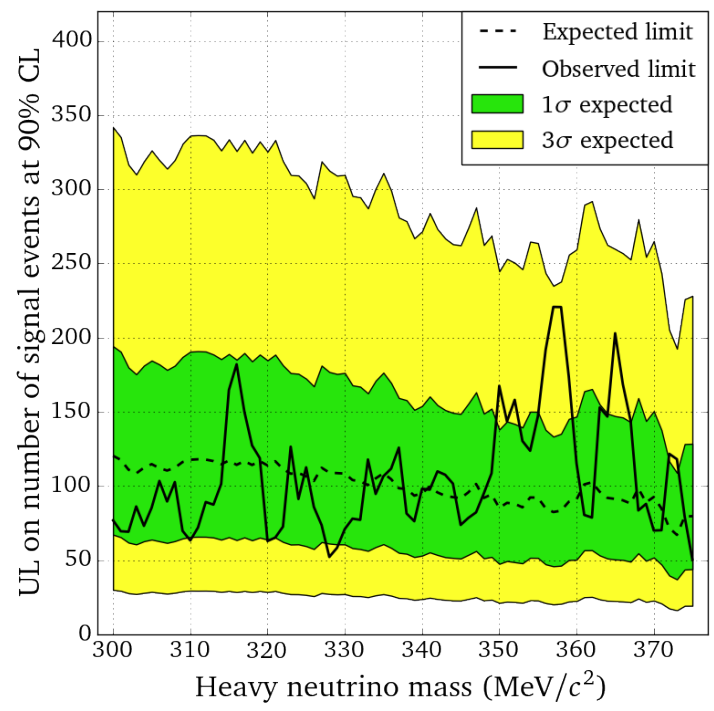

(b)

Figure 1: (a) Missing mass distribution of data and background estimate in the signal and control regions. Error bars are data statistical errors. The lower plot shows the total uncertainty on the background estimate. (b) Expected and observed limits (at 90\% CL) on the number of expected $K^{+} \rightarrow \mu^{+} v_{h}$ events at each tested mass. No excess above $3 \sigma$ is observed.

with single $K^{+}$beam are used to set the limits. Periods with single $K^{-}$beam, where the beam halo background is higher, are used to study the background from beam halo muons.

\subsection{UL on the production branching ratio $\mathscr{B}\left(K^{+} \rightarrow \mu^{+} v_{h}\right)$}

In the decay $K^{+} \rightarrow \mu^{+} N$, the neutrino missing mass can be reconstructed as $m_{h}=\sqrt{m_{\text {miss }}^{2}}=$ $\sqrt{\left(p_{K}-p_{\mu}\right)^{2}}$. The kaon four-momentum $p_{K}$ is the nominal beam kaon one, measured from $K^{+} \rightarrow \pi^{+} \pi^{+} \pi^{-}$samples at regular intervals ( $\sim 500$ bursts). The muon four-momentum $p_{\mu}$ is that of a charged track reconstructed in the spectrometer, assuming muon mass. The acceptance and resolution of the $K^{+} \rightarrow \mu^{+} v_{h}$ channel are studied using dedicated $v_{h}$ MC simulations as a function of the $m_{h}$ between 240 and $380 \mathrm{MeV} / c^{2}$ at $1 \mathrm{MeV} / c^{2}$ intervals. The observed and expected $m_{m i s s}$ spectra are compared to set limits on the number of observed $K^{+} \rightarrow \mu^{+} v_{h}$ decays for each tested $m_{h}$. There limits are translated into limits on the heavy neutrino production branching ratio $\mathscr{B}\left(K^{+} \rightarrow \mu^{+} v_{h}\right)$ and on the mixing matrix element $\left|U_{\mu 4}\right|^{2}$.

The event selection was based on one positively charged muon track with no cluster of energy deposition with $E>2 \mathrm{GeV}$ associated to the track. Five-dimensional cuts in the $\left(z_{v t x}, \theta, p, c d a, \phi\right)$ space are applied to suppress background from beam halo muons. Here the $z_{v t x}$ is the longitudinal position of the reconstructed vertex, $\theta$ is the angle between $K^{+}$and $\mu^{+}$direction, and $\phi$ the azimuthal angle of the muon in the transverse plane. The signal region $300 \mathrm{MeV} / c^{2}<m_{h}<$ $375 \mathrm{MeV} / c^{2}$ is used for the search. The main background components are shown on Figure I, dominated by $K^{+} \rightarrow \pi^{0} \mu^{+} v_{\mu}$ with the $\pi^{0}$ undetected. The total number of kaon decays in the 


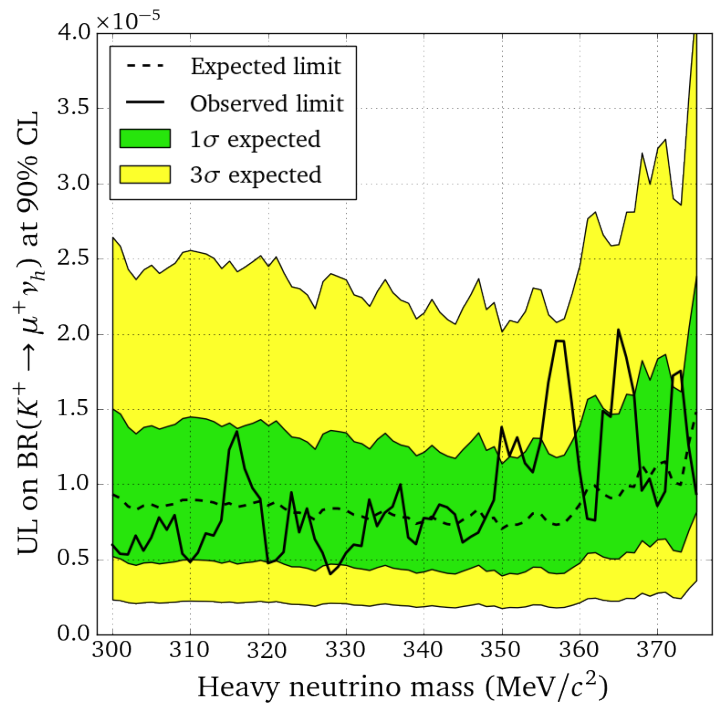

(a)

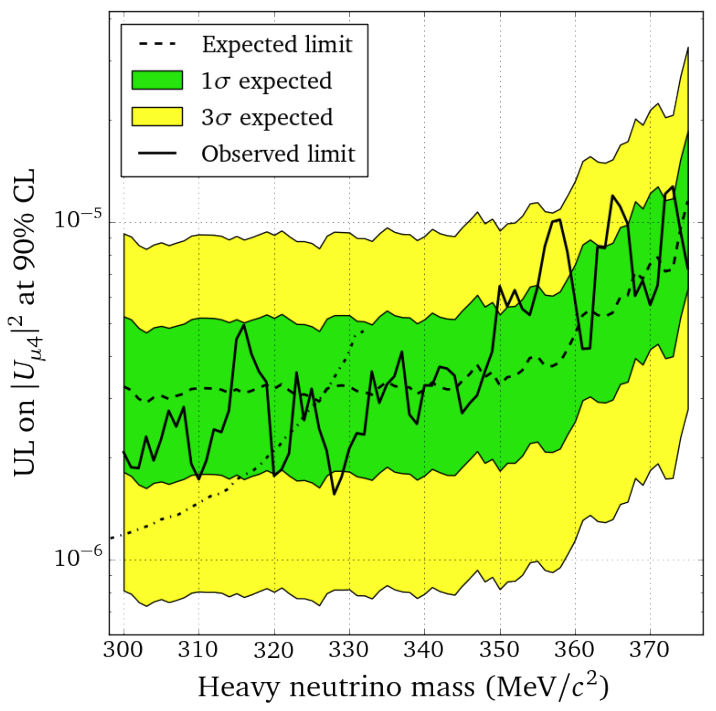

(b)

Figure 2: Expected and observed upper limits (at 90\% CL) on the branching ratio of $K^{+} \rightarrow \mu^{+} v_{h}$ (a) and the mixing matrix element $\left|U_{\mu 4}\right|^{2}$ (b) at each tested mass. The existing limit from KEK E089 [四] is also shown. Below $300 \mathrm{MeV} / c^{2}$ there is a limit of $\mathscr{O}\left(10^{-8}\right)$ from E979 [B]].

fiducial volume is $N_{K}=6 \times 10^{7}$, taking into account the trigger downscaling of 150 . More details on analysis procedure can be found [ $[\mathbb{8}]$

A peak search of the $m_{\text {miss }}$ distribution in steps of $1 \mathrm{MeV} / c^{2}$ is performed. For each heavy neutrino mass $m_{h}$ considered, a window of size $\pm \sigma_{h}=120 \mathrm{MeV} / c^{2}-0.03 \cdot m_{h}$ is used, corresponding to the resolution on the heavy neutrino mass. The upper limits at $90 \%$ confidence level (CL) on the number of reconstructed $K^{+} \rightarrow \mu^{+} v_{h}$ events $n_{U L}$ are computed by applying the RolkeLopez method $[\mathbb{}]$ for the case of a Poisson process in presence of gaussian background. The input necessary to the computation are the number of observed data events, the number of expected background events, and their uncertainties. The local significance never exceed $3 \sigma$ and therefore, no signal is observed. The obtained limits on the branching ratio are shown in Figure $\square$ together with the translated upper limits on the mixing matrix element $\left|U_{\mu 4}\right|^{2}$.

\section{Status of the NA62 experiment}

\subsection{The $K^{+} \rightarrow \pi^{+} v \bar{v}$ decay in the Standard Model}

The $K^{+} \rightarrow \pi^{+} v \bar{v}$ decay is a flavour changing neutral current process proceeding through box and electroweak penguin diagrams. The process is very rare, due to quadratic GIM mechanism and strong Cabibbo suppression. The dominant contribution comes from the short-distance physics of the top quark loop, with a small charm quark contribution and long-distance corrections. This makes the $K^{+} \rightarrow \pi^{+} v \bar{v}$ very clean theoretically and sensitive to physics beyond the SM, probing the highest mass scales among the rare meson decays [], 미, [13, [4], ㅍ]. The standard model 


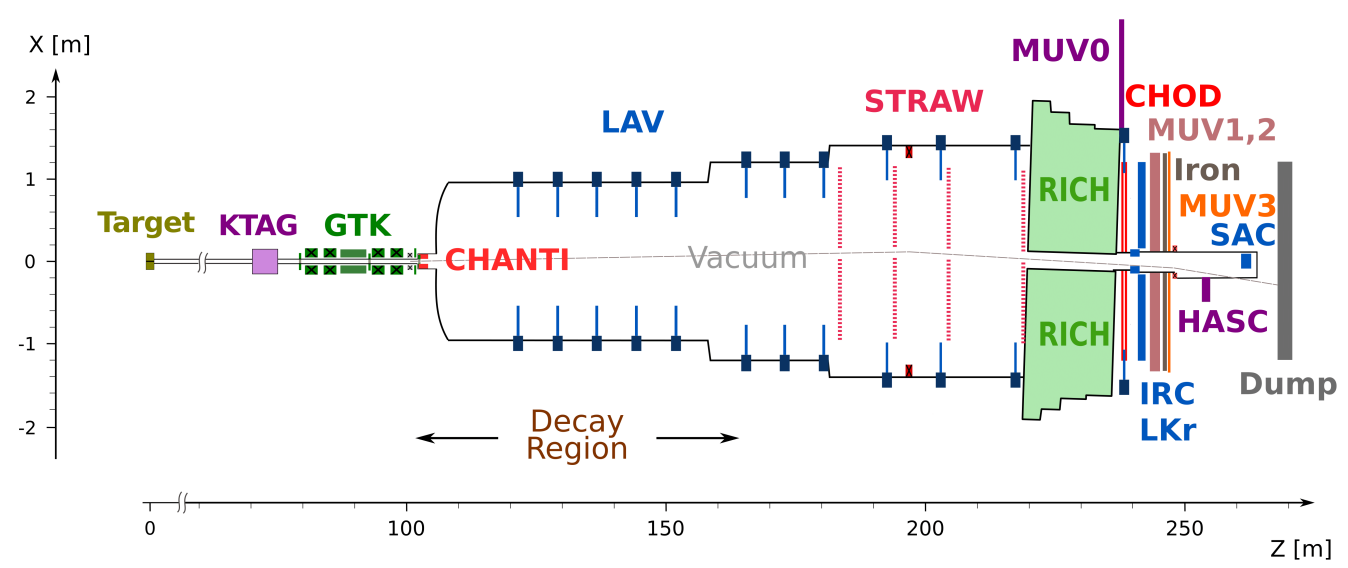

Figure 3: Schematic layout of the NA62 experiment in the $x z$ plane

prediction using elements of the Cabibbo-Kobayashi-Maskawa (CKM) matrix extracted from treelevel processes is [9, [0]].

$$
B R\left(K^{+} \rightarrow \pi v \bar{v}\right)=(8.4 \pm 1.0) \times 10^{-11}
$$

The knowledge of the external inputs dominate the uncertainties on the predictions. The experimental result [ए6]

$$
B R\left(K^{+} \rightarrow \pi v \bar{v}\right)=\left(17.3_{-10.5}^{+11.5}\right) \times 10^{-11},
$$

was obtained using stopped kaons. The result is $\sim 1 \sigma$ away from the SM expectation, but the measurement was based on only few events and the experimental uncertainties are large.

\subsection{NA62 detector setup}

The fixed target NA62 experiment aims at measuring the branching ratio of the $K^{+} \rightarrow \pi^{+} v \bar{v}$ decay with $10 \%$ precision. A sample of about $10^{13}$ kaon decays should be collected in few years of data-taking using the $400 \mathrm{GeV} / c$ primary SPS proton beam. A maximum of $10 \%$ of background contamination is required, necessitating a background rejection factor of the order of $10^{12}$. The beam impinges on a beryllium target producing secondary particles, of which the kaon component is $6 \%$. A $100 \mathrm{~m}$ long beam line selects, collimates, focuses and transports charged particles of (75.0 \pm 0.8$) \mathrm{GeV} / c$ momentum to the evacuated fiducial decay volume.

Figure [3] shows the experimental apparatus in operation since 2014. The KTAG is a differential Cherenkov detector filled with $N_{2}$ placed in the beam to identify and timestamp kaons. It is followed by the Gigatracker (GTK), three silicon pixel stations of $6 \times 3 \mathrm{~cm}^{2}$ surface exposed to the full $750 \mathrm{MHz}$ beam rate. It is used to timestamp and measure the momentum of the beam particles before entering the vacuum region downstream. The CHANTI detector placed after the Gigatracker tags hadronic interactions in the last GTK station. The magnetic spectrometer made of four straw chambers and a dipole magnet between the second and third chamber is used to measure the momentum of downstream charged particles. It is followed by a $17 \mathrm{~m}$ long RICH counter filled with $N e$, used to separate $\pi^{+}, \mu^{+}$and $\mathrm{e}^{+}$. The time of charged particles is measured both with the RICH and with an array of scintillator (CHOD) located downstream of the RICH. Two hadronic 


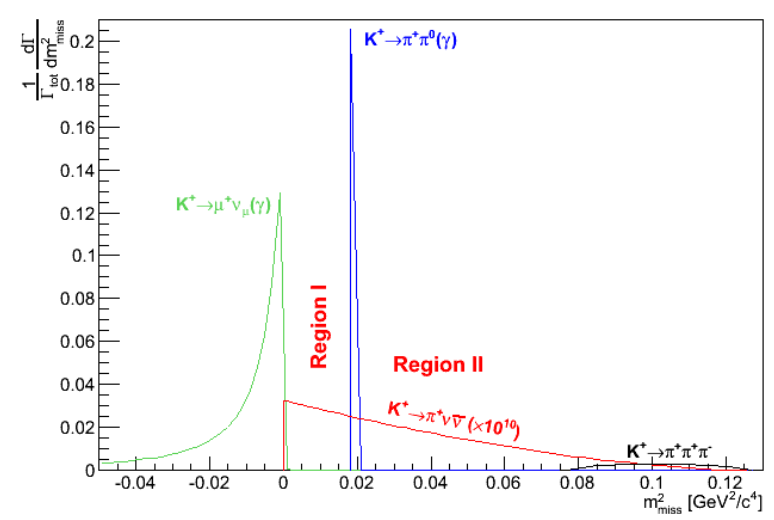

(a)

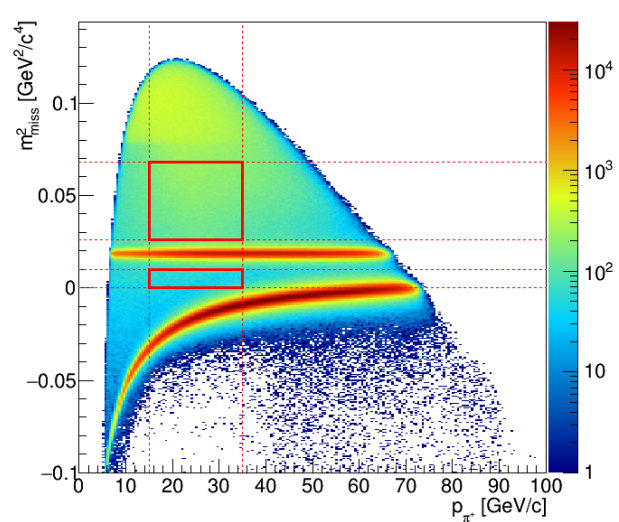

(b)

Figure 4: (a) $m_{\text {miss }}^{2}$ shapes for signal and backgrounds of the main $K^{+}$decay modes: the backgrounds are normalized according to their branching ratio; the signal is multiplied by a factor $10^{10}$ (b) Distribution of $m_{\text {miss }}^{2}$ as a function of track momentum for events selected on minimum bias data; The bands corresponding to $K^{+} \rightarrow \pi^{+} \pi^{0}$ and $K^{+} \rightarrow \mu^{+} v_{\mu}$ decays are clearly visible; the signal regions (red box) are drawn for reference.

calorimeters (MUV1 and MUV2) and a fast scintillator array (MUV3) provide further separation between $\pi^{+}$and $\mu^{+}$. A set of photons vetoes (LAVs, LKr, IRC, SAC) hermetically cover angles up to $50 \mathrm{mrad}$ to reject extra electromagnetic activity. A detailed description of the apparatus and its performances in 2015 can be found in [ए7]].

\subsection{The $K^{+} \rightarrow \pi^{+} v \bar{v}$ analysis}

The analysis of $5 \%$ of the 2016 dataset corresponding to $2.3 \times 10^{10}$ kaons is presented here. The $\pi v \bar{v}$ signature is one track in the initial and final state with two missing neutrinos. The main kinematic variable is $m_{\text {miss }}^{2} \equiv\left(P_{K}-P_{\pi^{+}}\right)^{2}$, where $P_{K}$ and $P_{\pi^{+}}$are the 4-momenta of the $K^{+}$and $\pi^{+}$respectively. The theoretical shape of the main $K^{+}$background decay modes are compared to the $K^{+} \rightarrow \pi^{+} v \bar{v}$ on Figure $\theta$. The analysis is done in the $\pi^{+}$momentum range between 15 and $35 \mathrm{GeV} / c$ to leave at least $40 \mathrm{GeV}$ of missing energy. Two regions are used: region 1 between $K^{+} \rightarrow \mu^{+} v_{\mu}\left(K_{\mu 2}\right)$ and $K^{+} \rightarrow \pi^{+} \pi^{0}\left(K_{2 \pi}\right)$ and region 2 between $K_{2 \pi}$ and $K^{+} \rightarrow \pi^{+} \pi^{+} \pi^{-}\left(K_{3 \pi}\right)$ (See Figure $\left(\right.$ ). The main backgrounds entering those regions are $K_{\mu 2}$ and $K_{2 \pi}$ decays through non gaussian resolution and radiative tails; $K_{3 \pi}$ through non gaussian resolution; $K^{+} \rightarrow \pi^{+} \pi^{-} e^{+} v_{e}\left(K_{e 4}\right)$ and $K^{+} \rightarrow l^{+} \pi^{0} v_{l}\left(K_{l 3}\right)$ by not detecting the extra $\pi^{-}, e^{+}, \pi^{0}$ particles. Another important source of background is the "beam related" background coming from upstream decays and beam-detector interactions. Each of the background processes requires different rejection procedure depending on its kinematics and type of charged particle in the final state. The main requirements for the analysis are: excellent kinematic reconstruction to reduce kinematic tails; precise timing to reduce the kaon mis-tagging probability; no extra in-time activity in all of the electromagnetic calorimeters to suppress $\pi^{0} \rightarrow \gamma \gamma$ decays (photon rejection); clear separation between $\pi / \mu / e$ tracks to suppress decays 


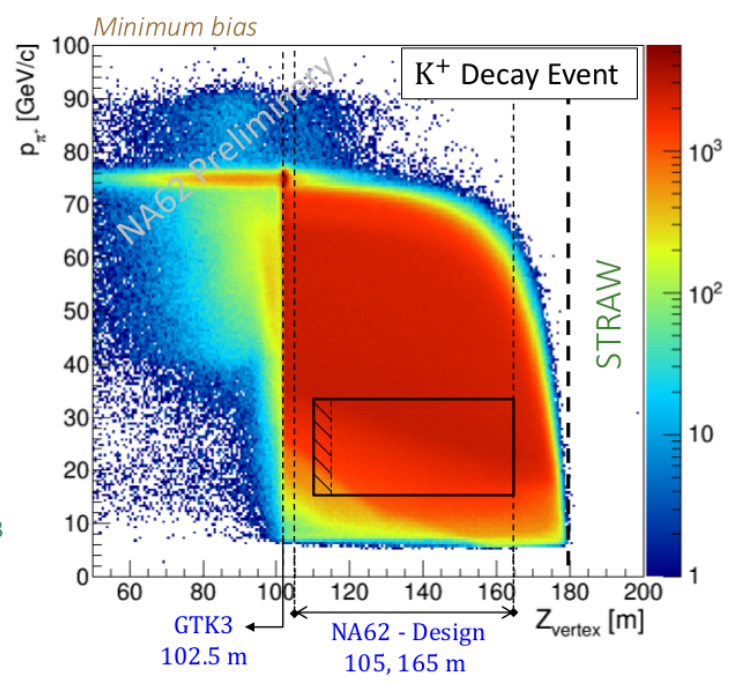

(a)

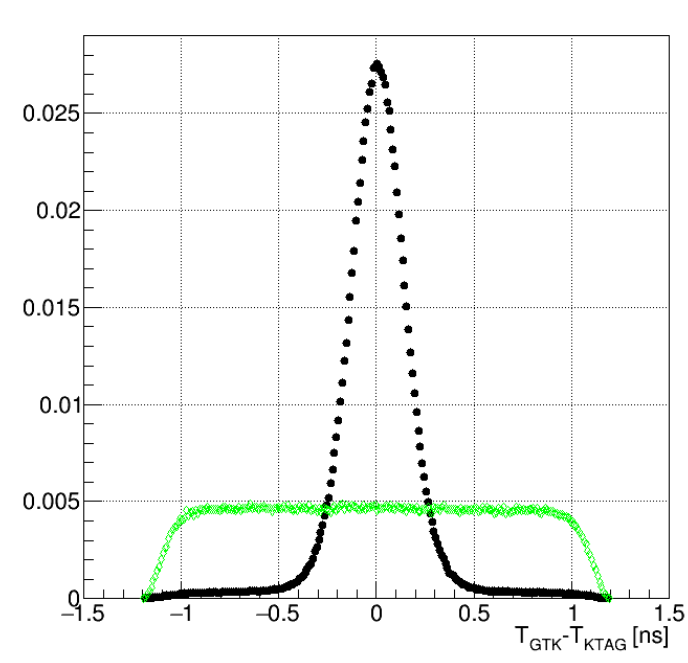

(b)

Figure 5: (a) $z$ position of the reconstructed kaon decay vertex vs $\pi^{+}$momentum; the fiducial region is marked by a solid rectangle and varies depending on the position of the $\pi^{+}$in the first STRAW chamber (black solid dashed line). (b) Distribution of the time difference between GTK and KTAG time candidates for $K^{+}$and $\pi^{+}$selected from $K^{+} \rightarrow \pi^{+} \pi^{+} \pi^{-}$events (black dots); green dots are the same quantities computed with a random beam track instead of $K^{+}$track;

with $\mu^{+}$or $e^{+}$in the final state (particle identification); low multiplicity cuts in the downstream detectors are used to further suppress decays with multiple charged tracks in the final state.

Events with single $\pi^{+}$topology are selected using the STRAW, CHOD and RICH detectors. The $\pi^{+}$track must have a matching pair of slabs in the CHOD and a reconstructed ring in the RICH, where the time is measured with $100 \mathrm{ps}$ resolution. The parent $\mathrm{K}^{+}$track is reconstructed and timestamped in GTK with 100 ps resolution as shown on Figure [1. Both the pion and the kaon must have in-time signal in the KTAG. A kaon decay event is then created with a decay vertex defined by the intersection of the GTK and STRAW tracks. The closest distance of approach between the GTK and STRAW tracks and the timing provide a precise $K^{+}-\pi^{+}$matching. The vertex spatial resolution is $\sigma \sim 1.5 \mathrm{~mm}$. The $K^{+}-\pi^{+}$mis-tagging probability is $\sim 1.7 \%$ at $40 \%$ of nominal intensity for signal acceptance of about $75 \%$.

All kaon decays within a $50 \mathrm{~m}$ fiducial region beginning $10 \mathrm{~m}$ downstream to the last GTK station (GTK3) are considered to reject events originating from interactions of beam particles in GTK and kaon decays upstream of GTK3 as shown on Figure [5. The kinematics of the selected events can be seen in Figure $\$$ (b). Signal region definitions are driven by the $m_{\text {miss }}^{2}$ resolution. Kinematic tails due to track mis-reconstruction of $K^{+} \rightarrow \mu^{+} v_{\mu}, K^{+} \rightarrow \pi^{+} \pi^{0}$ and $K^{+} \rightarrow \pi^{+} \pi^{+} \pi^{-}$decays set the level of background in the signal regions. To reduce it, 3D signal regions are constructed, defined by the standard $m_{m i s s}^{2}$, the same quantity computed by replacing the STRAW momentum with that measured by the RICH under a $\pi^{+}$mass hypothesis $\left(m_{m m i s s}^{2}(R I C H)\right)$ and computed by using nominal $K^{+}$momentum $\left(m_{m m i s s}^{2}(N o-G T K)\right)$. The kinematic suppression, defined as the probabil- 


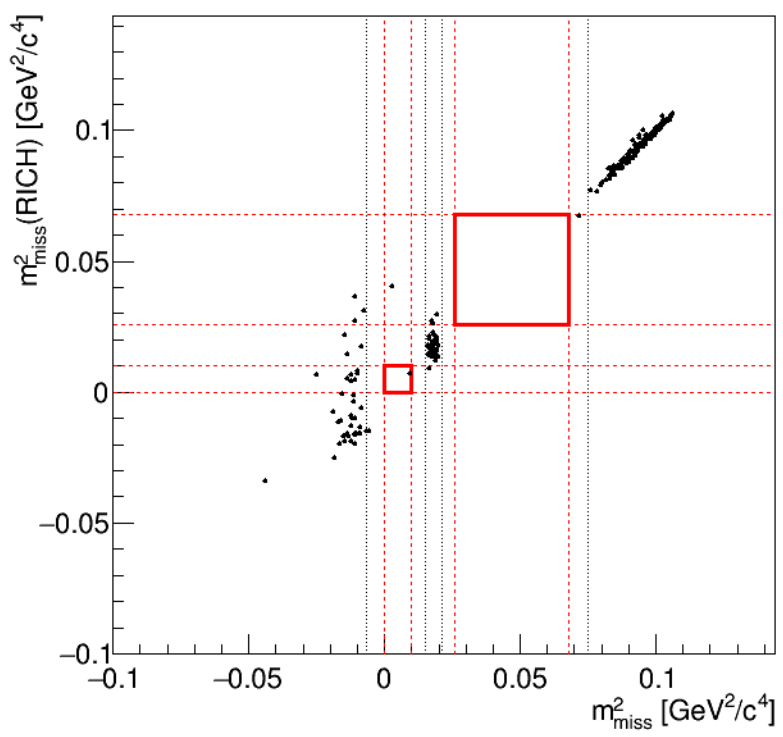

(a)

Figure 6: Distribution of $\left(m_{m i s s}^{2}(R I C H), m_{m i s s}^{2}\right)$ plane of $K^{+} \rightarrow \pi^{+} v \bar{v}$-triggered events passing the selection, except for the cut on $m_{\text {miss }}^{2}(N o-G T K)$; signal regions (red solid line boxes) and background region boxes (dashed lines) are drawn for reference; the event in region 1 has $m_{\text {miss }}^{2}$ (No$-G T K$ ) outside the signal region.

ity of the $K^{+} \rightarrow \mu^{+} v_{\mu}\left(K^{+} \rightarrow \pi^{+} \pi^{0}\right)$ decays to enter the 3D signal regions is $3 \times 10^{-4}\left(6 \times 10^{-4}\right)$. It is measured using $K^{+} \rightarrow \mu^{+} v_{\mu}$ and $K^{+} \rightarrow \pi^{+} \pi^{0}$ events selected using $\pi v \bar{v}$-like selection on a control triggered data sample.

The separation of $\pi^{+}, \mu^{+}$and $e^{+}$is achieved with the calorimeters and the RICH. Multivariate technique using the released energy, the energy sharing between the calorimeters and the shape of the deposited energy provides $10^{5}$ muon suppression for $80 \% \pi^{+}$efficiency. A combination of RICH parameters are further used to obtain another factor of $10^{2}$ suppression for a $\pi^{+}$efficiency of $80 \%$. The two methods are independent thus providing 7 orders of magnitude overall muon suppression and $60 \% \pi^{+}$efficiency. The performances are measured on kinematically selected $K^{+} \rightarrow \pi^{+} \pi^{0}$ and $K^{+} \rightarrow \mu^{+} v_{\mu}$ decays on control triggered data.

Left over events after $\pi^{+}$identification events are mainly $K^{+} \rightarrow \pi^{+} \pi^{0}$, which are further suppressed by rejecting in-time coincidences between the $\pi^{+}$and energy deposits in the electromagnetic calorimeters LKr, LAVs, SAC, IRC. The resulting $\pi^{0}$ suppression is $(1.2 \pm 0.2) \times 10^{-7}$, as measured from minimum bias and $K^{+} \rightarrow \pi^{+} v \bar{v}$ triggered events before and after $\gamma$ rejection, respectively. Accidental losses are measured using control triggered $K^{+} \rightarrow \mu^{+} v_{\mu}$ events and are in the $15-20 \%$ range for $40-50 \%$ of the nominal intensity.

A sample of $K^{+} \rightarrow \pi^{+} \pi^{0}$ control triggered minimum bias data is used for normalization. About $0.064 K^{+} \rightarrow \pi^{+} v \bar{v}$ events are expected over $2.3 \times 10^{10} K^{+}$decays. The distribution of the remaining events in the $2 \mathrm{D}$ plane of $m_{m i s s}^{2}$ vs $m_{m i s s}^{2}(R I C H)$ is shown on Figure 田. Background 
expectations from $K^{+} \rightarrow \pi^{+} \pi^{0}, K^{+} \rightarrow \mu^{+} v_{\mu}$ and $K^{+} \rightarrow \pi^{+} \pi^{+} \pi^{-}$are $0.024,0.011$ and 0.017 respectively. They are estimated directly from events outside the signal regions, with the measured kinematic tails used for extrapolation in the signal regions. Simulations studies indicate that backgrounds from other processes are lower or negligible. The analysis is still on-going together with an optimization of the selection to further reduce backgrounds and increase signal acceptance. No events are observed in signal regions. NA62 aims to reach the SM sensitivity from the analysis of the 2016 data and to select few tens of $K^{+} \rightarrow \pi^{+} v \bar{v}$ events from the analysis of the data taken in 2017 and expected in 2018.

\section{Conclusions}

Using the data collected in 2007, a peak search in the mass spectrum of the $K^{+} \rightarrow \mu^{+} \nu_{\mu}$ decay has been performed in of $1 \mathrm{MeV} / c^{2}$. New limits on the heavy neutrino production are set at the level of $10^{-5}-10^{-6}$ on the mixing matrix element $\left|U_{\mu 4}\right|^{2}$ in the range $300-375 \mathrm{MeV} / \mathrm{c}^{2}$. The commissioning of the NA62 experiment in 2015 [प]] shows that all performances are in line with the requirements to perform the full scale $\pi \nu \bar{v}$ analysis. The first $K^{+} \rightarrow \pi^{+} v \bar{v}$ data sample has been collected in 2016. No event was found after the analysis of $2.3 \times 10^{10} \mathrm{~K}^{+}$decays corresponding to 5 $\%$ of the full 2016 dataset. Using the full 2016 dataset $\mathscr{O}(1)$ SM events are expected. Optimizations are ongoing to increase the signal acceptance and to reduce the signal-to-background ratio. The NA62 will continue to take data during 2017 and 2018.

\section{References}

[1] T. Asaka, M. Shaposhnikov, Phys. Lett. B 620, 17 (2005)

[2] R. Schrock, Phys. Lett. B 96, 159 (1980)

[3] A. V. Artamonov et al., Phys. Rev. D 91, 052001 (2015)

[4] R. S. Hayano et al., Phys. Rev. Lett. 49, 1305 (1982)

[5] The NA62 Collaboration, Phys. Lett. B 719, 326 (2013)

[6] The NA62 Collaboration, Eur. Phys. J. C 52, 875 (2007)

[7] W. A. Rolke, A. M. Lopez, Nucl. Instrum. Methods A 458, 754 (2001)

[8] The NA62 Collaboration, Phys. Lett. B 772, 712-718 (2017)

[9] A. J. Buras, D.Buttazzo, K.Girrbach-Noe, R. Knegjens, JHEP 11, 033 (2015)

[10] J. Brod, M. Gorbahn, E. Stammou, Phys. Rev. D 83, 034030 (2011)

[11] M. Blanke, A. J. Buras, B. Duiling, K. Gemmler, S. Gori, JHEP 903, 108 (2009)

[12] A. J. Buras, D. Buttazzo, R. Knegjens, JHEP 1511, 166 (2015)

[13] T. Blazek, P. Matak, Int. J. Mod. Phys. A 29.no.27, 1450162 (2014)

[14] Isidori et al., JHEP 0608, 064 (2006)

[15] M. Blanke, A. J. Buras, S. Recksiegel, Eur. Phys. J. C 76.no.4, 182 (2016)

[16] A. V. Artamonov et al. (E949 Collaboration), Phys. Rev. D 79, 092004 (2009)

[17] The NA62 Collaboration, JINST 12, P05025 (2017) 